\title{
Sustainability of Intraocular Pressure Reduction of Travoprost Ophthalmic Solution in Subjects with Normal Tension Glaucoma
}

\author{
Tomoko Naito · Shinichi Okuma · Mikio Nagayama · Shiro Mizoue • \\ Mineo Ozaki · Koji Namiguchi · Kazuhisa Miyamoto · Masaki Tanito • \\ Keiji Yoshikawa
}

To view enhanced content go to www.advancesintherapy.com Received: December 20, 2015 / Published online: February 9, 2016

(C) The Author(s) 2016. This article is published with open access at Springerlink.com

\section{ABSTRACT}

Introduction: We examined the sustainability of the intraocular pressure (IOP)-lowering efficacy of travoprost $(0.004 \%)$ ophthalmic solution in subjects with normal tension glaucoma (NTG).

Methods: Travoprost ophthalmic solution was given once daily at $9 \mathrm{PM}$ to subjects with newly

T. Naito and S. Okuma contributed equally to the manuscript.

Electronic supplementary material The online version of this article (doi:10.1007/s12325-016-0297-6) contains supplementary material, which is available to authorized users.

T. Naito $(\square)$

Department of Ophthalmology, Okayama

University Graduate School of Medicine,

Shikata-cho, Kita-ku, Okayama, Japan

e-mail: tomokoum@gmail.com

S. Okuma - K. Namiguchi · K. Miyamoto

Sumitomo Besshi Hospital, Oji-cho, Niihama,

Ehime, Japan

M. Nagayama

Nagayama Eye Clinic, Goban-cho, Kasaoka,

Okayama, Japan

S. Mizoue - K. Namiguchi

Department of Ophthalmology, Ehime University

Graduate School of Medicine, Shitsukawa, Toon,

Ehime, Japan diagnosed NTG or with NTG who had not received any ocular hypotensives within the previous 30 days. IOP was measured at three time points (9 AM, $1 \mathrm{PM}$, and $5 \mathrm{PM}$ ) at baseline and week 12 visits, and at one time point (9 AM) at week 4 and week 8 visits. Conjunctival hyperemia, superficial punctate keratopathy, and other adverse events were evaluated during the observation period.

Results: Thirty subjects (12 males and 18 females; mean age 65.6 years) from 32 subjects enrolled were included in the efficacy analysis. The mean IOPs ( \pm standard deviation) of $16.6 \pm 1.4,15.7 \pm 1.8$, and $15.7 \pm 2.2 \mathrm{mmHg}$ at $9 \mathrm{AM}, 1 \mathrm{PM}$, and $5 \mathrm{PM}$, respectively, at

M. Ozaki

Ozaki Eye Hospital, Kamezaki, Hyuga, Miyazaki, Japan

\section{Tanito}

Department of Ophthalmology, Shimane University Faculty of Medicine, 89-1 Enya, Izumo, Shimane, Japan

\section{Tanito}

Division of Ophthalmology, Matsue Red Cross

Hospital, 200 Horomachi, Matsue, Shimane, Japan

K. Yoshikawa

Yoshikawa Eye Clinic, Nakamachi, Machida, Tokyo, Japan 
baseline reduced significantly to the mean IOPs of $13.0 \pm 1.8,12.7 \pm 1.8$, and $12.8 \pm 1.6 \mathrm{mmHg}$, respectively, at week $12(P<0.0001$ for every time point). Together with the mean IOPs of $13.4 \pm 1.9 \mathrm{mmHg}$ at week 4 and $13.2 \pm 1.9 \mathrm{mmHg}$ at week 8 , the pooled IOP during the observation period for up to 12 weeks showed a statistically and clinically significant reduction of IOP at $9 \mathrm{AM}$. (3.4 $\mathrm{mmHg}$ or $20.3 \%$ reduction from baseline, $P<0.0001)$. There were no adverse events leading to treatment discontinuation.

Conclusion: This multi-center collaborative study suggests that IOP-lowering efficacy of travoprost ophthalmic solution persists during the day at the clinically relevant level in subjects with NTG.

Funding: Alcon Japan Ltd.

Trial registration: University Hospital Medical Information Network, UMIN ID: 000011621.

Keywords: Intraocular pressure (IOP); IOP-lowering; Normal tension glaucoma; Sustainability; Travoprost

\section{INTRODUCTION}

The Tajimi epidemiological study reported that the prevalence of glaucoma in those aged 40 years and older in Japan is $5 \%$, and primary open-angle glaucoma accounts for $78 \%$ of the patients; more than $90 \%$ of these glaucoma patients were diagnosed as normal tension glaucoma (NTG) [1, 2]. Currently, the only proven treatment for glaucoma is to lower intraocular pressure (IOP), the major risk factor for progression of glaucomatous visual field damage [3-5]. It has been reported that a similar correlation between decreased IOP and progression of visual field defect has been noted for NTG [6]. In cases with NTG, as an initial treatment, it is recommended that IOP be lowered by $20 \%$ from baseline [7], since this level of IOP reduction prevents progression of a visual field defect during long-term follow-up $[7,8]$. Other than IOP level, IOP fluctuations defined as the differences between maximum and minimum IOP levels during follow-up periods may be another risk factor for visual field defects [9, 10] even in NTG [8]. Minimization of IOP fluctuations may therefore be clinically important.

Patients with NTG are usually treated with topical medication(s) to lower IOP. Among currently available ocular hypotensives, prostaglandin (PG) analogs are the first-line choice of agent for the treatment of NTG because of their strong IOP-lowering effect [11] and their benefits of compliance with fewer systemic side effects and less frequent dosing. Once-daily administration of PG analogs is based on their sustainability of IOP-lowering effect for almost an entire day. Sustained efficacy of PGs over more than $24 \mathrm{~h}$ may minimize the IOP fluctuation even if the daily administration time may differ to some degree. Travoprost ophthalmic solution, one of four currently available PG ocular hypotensives in Japan, was reported to have an IOP-lowering effect equal to or even superior than $0.005 \%$ latanoprost eye drops $\left(\right.$ Xalatan $^{\circledR}$; Pfizer, New York, NY, USA) [12]. Other reports have shown that travoprost ophthalmic solution has an effect of sustainable IOP reduction for $24 \mathrm{~h}$ or longer [13-17], and is thus expected to lessen IOP fluctuations. However, to date, only a few single-site studies have investigated the IOP-lowering effects of travoprost in respect to its sustainability and IOP fluctuation $[11,18$, 19]. We therefore conducted multi-centered studies in subjects with Japanese NTG patients. Ocular hypotensives can affect the ocular 
surface mainly because of their preservative agent benzalkonium chloride (BAC) [20]. A BAC-free formulation of travoprost, Travatan $\mathrm{Z}^{\circledR} \quad(0.004 \%$ travoprost ophthalmic solution; Alcon Laboratories, Inc., Fort Worth, TX, USA) [21], contains a zinc-based self-preservation system (sofZia ${ }^{\mathrm{TM}}$; Alcon Laboratories, Inc., Fort Worth, TX, USA), which would be expected to have a minimal effect on the ocular surface [20]. To verify this, we assessed ocular adverse events including superficial punctate keratopathy (SPK) and conjunctiva hyperemia following treatment with travoprost $0.004 \%$ in subjects with newly diagnosed NTG.

\section{METHODS}

\section{Subjects}

We enrolled 32 outpatients from six institutions (Okayama University, Nagayama Eye Clinic, Minami-Matsuyama Hospital, Ozaki Eye Hospital, Sumitomo Besshi Hospital, and Shimane University) between September 2013 and June 2014. Subjects with newly diagnosed NTG or with NTG who had not received any IOP-lowering ophthalmic solutions within the previous 30 days, received travoprost $(0.004 \%)$ ophthalmic solution for 3 months to evaluate the efficacy and safety of this treatment. Subjects with at least one eye that met the study criteria were enrolled.

The inclusion criteria included: (1) age 20 years or more at the time of written informed consent obtained, (2) all IOPs measured at $9 \mathrm{AM}, 1 \mathrm{PM}$, and $5 \mathrm{PM}$ within the range of $>10$ and $<20 \mathrm{mmHg}$ in the study eye at baseline, (3) either male or female. There were no specific criteria regarding outpatient/inpatient treatment history. The exclusion criteria included: (1) pregnancy (or women who wished to become pregnant during the study period) or lactation, (2) angle grade in Shaffer classification of 2 or less, (3) advanced glaucoma with the visual field mean deviation of less than $-12 \mathrm{~dB}$, (4) history of chronic or recurrent uveitis, scleritis, or herpes keratitis, (5) history of ocular trauma, intraocular surgery, or laser surgery in the study eye, (6) ocular infection or other ocular pathology other than glaucoma, (7) best-corrected visual acuity of $<0.2$, (8) difficulty in conducting applanation tonometry in the study eye, (9) history of hypersensitivity to PG analogs or any ingredients used in the study, (10) requirement for IOP-lowering drug(s) other than $0.004 \%$ travoprost ophthalmic solution, or oral carbonic anhydrase inhibitor during the study period, (11) requirement for any steroid therapy during the study period, (12) subjects who had received IOP-lowering ophthalmic solution within the past 30 days, or (13) subjects regarded as inappropriate for study enrollment as judged by the investigators. If both eyes were eligible, the study eye was determined as the eye with higher IOP at 9 AM of baseline. If bilateral IOP values were the same at $9 \mathrm{AM}$, the eye with higher IOP at $1 \mathrm{PM}$ of baseline was defined as the study eye. If bilateral IOP values were the same at $1 \mathrm{PM}$, the eye with higher IOP at $5 \mathrm{PM}$ of baseline was defined as the study eye. If all IOP values at 9 AM, 1 PM, and $5 \mathrm{PM}$ of baseline were equal, the right eye was defined as the study eye.

\section{Procedures}

Before starting the study, Alcon Japan Ltd. drafted the protocol, including sample size estimation, and fixed the protocol following discussion with a medical advisor (K.Y.). The protocol was approved by the institutional ethical review committees at Okayama University, Shimane University, Sumitomo 
Besshi Hospital, and Minami-Matsuyama Hospital. Because of the absence of institutional review committees at Ozaki Eye Hospital and Nagayama Eye Clinic, the committee of Asano Clinic (Saitama Japan) reviewed and approved the protocol for these sites. The study was registered and disclosed in UMIN (University Hospital Medical Information Network, UMIN ID: 000011621) before the first subject was included. Candidates who met the study criteria were thoroughly instructed in the contents and information of the ethical principles that have their origin in the tenets of the Declaration of Helsinki and ethical guidelines for clinical studies (fully revised on July 31, 2008). Subsequently, subjects who endorsed the informed consent form underwent baseline assessments, including IOP measurement, anterior segment examinations including slitlamp biomicroscopy and gonioscopy, central corneal thickness measurements by specular microscopy, and visual field testing using Humphrey Visual Field Analyzer (Carl Zeiss Japan, Tokyo, Japan) 30-2 SITA standard algorism, and those subjects who completed the baseline tests were enrolled in the study.

The enrolled subjects instilled one drop of travoprost $0.004 \% \quad$ (Travatan $Z^{\circledR} ;$ Alcon Laboratories, Inc., Fort Worth, TX, USA) into the conjunctival sac at $9 \mathrm{PM}$, once daily for 12 weeks. On each visit at week 4, 8, and 12 after starting therapy, IOP was measured and the degrees of SPK and conjunctival hyperemia were examined, and subjects were then interviewed regarding the presence of side effects. The same investigator at each institution measured IOP twice with a Goldmann applanation tonometer; the mean IOP of two measurements was used for the analyses. At baseline and week 12, IOP was measured at three time points: at 9 AM, 1 PM, and $5 \mathrm{PM}$, and at weeks 4 and 8 , IOP was measured at $9 \mathrm{AM}$. For each measurement, a range of $\pm 30 \mathrm{~min}$ from the scheduled time was allowed. Throughout the study period, the same investigator measured IOP for each subject.

For evaluation of SPK, the cornea was stained with fluorescein and observed with a slitlamp microscope. The National Eye Institute grid [22] was used to grade corneal staining in each of five zones (superior, temporal, inferior, nasal, and central) with a five-point scale (0-4) [23]. The mean SPK score was obtained by averaging scores among the five zones. Conjunctival hyperemia was scored according to four grades: 0, no vasodilatation; 1 , vasodilation of mainly small blood vessels; 2 , vasodilation of both large and small blood vessels; 3, marked vasodilation including both large and small blood vessels [24]. Reference photographs were used to grade the SPK and hyperemia. Periocular changes including eyelash, pigmentation, and sunken eyelid as compared with baseline were scored by 3-point categorical rating; scores 0 (not present), 1 (mild), and 2 (apparent). If at least one SPK score of five zones was rated as 3 or more, or the conjunctival hyperemia score was rated 3 , this was reported as an adverse event. Clinically significant findings also were reported as an adverse event at any time for any medical reason at the discretion of investigators.

\section{Statistical Analyses}

In the study protocol, the effective sample size was set at 29 to detect $2.5 \mathrm{mmHg}$ IOP reduction with $90 \%$ power and two-sided $5 \%$ alpha by one-sample $t$ test assuming $4.0 \mathrm{mmHg}$ standard deviation (SD). Thirty-four subjects were to be enrolled assuming a 15\% drop-out rate. Case reports with concealed personal information were sent to Bell Medical Solutions Inc. (Tokyo, Japan) to be doubly checked for accuracy. Any 
undescribed item was reinvestigated. Data were digitalized by single entry method after determination of eligibility for the subjects who entered the study; the medical advisor (K.Y.) and representatives of Alcon Japan confirmed the eligibility. The database was then locked, and statistical analyses were performed by Bell Medical Solutions Inc.

Descriptive statistics (mean, SD, and subject) number of IOP value, IOP change from baseline $(\Delta \mathrm{IOP})$, and IOP percent change $(\% \Delta \mathrm{IOP})$ were presented at each evaluation point. Based on a two-way repeated-measures analysis of variance (ANOVA) model, which considers repeated measurements of each subject, least-squares means and the confidence intervals (CIs) of IOP, $\Delta \mathrm{IOP}$, and $\% \Delta \mathrm{IOP}$ were calculated. The $\Delta \mathrm{IOP}$ and $\% \Delta \mathrm{IOP}$ from baseline were assessed by $t$ test. The primary efficacy endpoint of this study was the least-square mean of $\Delta \mathrm{IOP}$ from baseline at 9 AM pooled over weeks 4,8 and 12, which corresponds to the mean of three time points. Two-sided significance level was set at 0.05. To assess the change in diurnal IOP fluctuation in each subject, we calculated the difference between the maximum and minimum IOP values for each subject (diurnal IOP fluctuation) at baseline and week 12 and used the $t$ test to compare diurnal IOP fluctuation before and after treatment. We evaluated the safety of travoprost, including the conjunctival hyperemia score, SPK score, and peripheral eye changes from baseline by $t$ test. Statistical software used in this study was SAS ${ }^{\circledR}$ version 9.2 (SAS Institute Inc., Cary, NC, USA). In this study, statistical tests ( $P$ values) other than primary analyses were presented to describe the safety and efficacy profile of travoprost, rather than to advocate statistical significance. The primary analysis was performed on the full analysis set (FAS) while the safety analysis was performed on the safety data set as described later.

\section{RESULTS}

Thirty-two subjects were enrolled into the study (three more than the target sample size). One subject discontinued because of hospital transfer after baseline tests. All 31 subjects who completed the study were included in the safety data sets. One subject who had prior ocular surgery was excluded meaning 30 subjects were included in the FAS. IOPs measured outside \pm 30 min of the scheduled time (7 measurements of 5 subjects; maximum deviation was $67 \mathrm{~min}$ ) or IOPs measured outside the scheduled date (5 measurements of 4 subjects; maximum deviation was 14 days) were not excluded from the FAS. The FAS therefore included 12 males and 18 females, and the mean age at baseline was 65.6 years (range 40-84 years); the baseline mean IOP values ranged between 15.7 and $16.6 \mathrm{mmHg}$ during the measurement time points (Table 1 ).

The mean of IOPs, $\Delta \mathrm{IOPs}$, and $\% \Delta \mathrm{IOPs}$ measured throughout the study were

Table 1 Subject baseline characteristics

\begin{tabular}{ll}
\hline Characteristics & Values \\
\hline Male, $n(\%)$ & $12(40.0 \%)$ \\
Female, $n(\%)$ & $18(60.0 \%)$ \\
Age, years & $65.6 \pm 11.1(40-84)$ \\
Baseline IOP, mmHg & \\
9 AM & $16.6 \pm 1.4(14.0-20.0)$ \\
1 PM & $15.7 \pm 1.8(12.0-19.5)$ \\
5 PM & $15.7 \pm 2.2(11.0-19.0)$ \\
Corneal thickness, $\mu \mathrm{m}$ & $516.5 \pm 25.9(462-578)$ \\
MD, dB & $-3.45 \pm 3.19(-11.62-0.70)$ \\
PSD, dB & $5.89 \pm 4.55(1.39-16.12)$ \\
\hline
\end{tabular}

Data are shown as mean \pm standard deviation, range $(\min -\max )$, unless otherwise stated

$d B$ decibels, $I O P$ intraocular pressure, $M D$ mean deviation, PSD pattern standard deviation 
Table 2 Summary of measured IOP

\begin{tabular}{|c|c|c|c|c|c|c|c|c|}
\hline \multirow[t]{2}{*}{ Time } & \multicolumn{3}{|c|}{ Baseline } & \multirow{2}{*}{$\begin{array}{l}4 \mathrm{w} \\
9 \mathrm{AM}\end{array}$} & \multirow{2}{*}{$\begin{array}{l}8 \mathrm{w} \\
9 \mathrm{AM}\end{array}$} & \multicolumn{3}{|l|}{$12 \mathrm{w}$} \\
\hline & $9 \mathrm{AM}$ & 1 PM & $\overline{5 \mathrm{PM}}$ & & & $9 \mathrm{AM}$ & 1 PM & $5 \mathrm{PM}$ \\
\hline$n$ & 30 & 30 & 30 & 30 & 30 & 30 & 30 & 30 \\
\hline IOP $(\mathrm{mmHg})$ & 16.6 & 15.7 & 15.7 & 13.4 & 13.2 & 13.0 & 12.7 & 12.8 \\
\hline SD & 1.43 & 1.82 & 2.17 & 1.85 & 1.90 & 1.79 & 1.75 & 1.62 \\
\hline$\Delta \mathrm{IOP}(\mathrm{mmHg})$ & - & - & - & -3.2 & -3.3 & -3.6 & -3.1 & -2.9 \\
\hline SD & - & - & - & 1.7 & 1.8 & 1.5 & 1.6 & 1.8 \\
\hline$\% \Delta \mathrm{IOP}(\%)$ & - & - & - & -19.2 & -20 & -21.6 & -19.2 & -17.9 \\
\hline SD & - & - & - & 10.2 & 10.5 & 8.9 & 8.9 & 10.1 \\
\hline
\end{tabular}

$\triangle I O P$ IOP change, \% $\triangle I O P$ IOP percent change, IOP intraocular pressure, $S D$ standard deviation, $w$ week

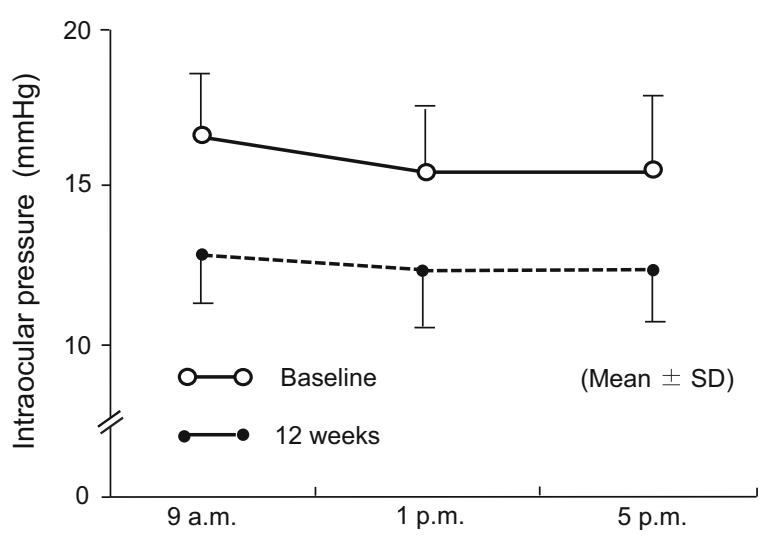

Fig. 1 Changes in diurnal intraocular pressure. $S D$ standard deviation

summarized in Table 2. For further clarification, the diurnal IOPs at baseline and week 12 are also shown in Fig. 1. The mean IOPs at all time points of every visit were lower than the respective IOPs at baseline. By comparison of least-squares mean of IOP changes based on repeated-measures ANOVA, the reductions in IOP after the start of topical travoprost were significant at all time points of IOP measured $(P<0.0001$ for all comparison pairs, $t$ test; Table 3). At $9 \mathrm{AM}$, the IOP was reduced by $3.4 \mathrm{mmHg}(P<0.0001)$ or $20.3 \%(P<0.0001)$ from baseline when the IOPs measured at weeks 4,8 , and 12 were pooled (Table 3 ). Also, the result from the per protocol set (PPS), the data set excluding IOP data which deviate from protocol guidelines as day/time allowance of IOP evaluation, was similar with FAS result. (PPS: At 9 AM, IOP was reduced by $3.1 \mathrm{mmHg}$ from baseline when the IOPs measured at weeks $4,8$, and 12 were pooled, $P<0.0001)$. We further analyzed the diurnal fluctuations in IOPs before and after travoprost treatment (Fig. 2). The diurnal IOP fluctuation of $2.2 \pm 1.4 \mathrm{mmHg}$ (mean $\pm \mathrm{SD}$ ) at baseline was reduced to $1.6 \pm 0.9 \mathrm{mmHg}$ at week 12 $(P=0.0333, \quad t \quad$ test $), \quad$ indicating a $0.6 \pm 1.5 \mathrm{mmHg}$ reduction.

Analysis of the safety data set showed that SPK scores did not deteriorate at any visits after the start of travoprost (Table 4); the maximum score reported in each corneal zone was 2 among the study eyes. Compared with the baseline value $(0.3 \pm 0.5)$, conjunctival hyperemia scores were statistically higher at weeks $4 \quad(0.6 \pm 0.6, \quad P=0.0169)$ and 12 $(0.6 \pm 0.7, P=0.0323)$. However, $21(70.0 \%)$ of the subjects did not have an increase in their score from the baseline value during the study period; no study eye was associated with an adverse event (score 3 or higher; Table 5). The changes in eyelash scores from baseline 
Table 3 Least-squares mean of IOP changes and percent IOP changes from baseline

\begin{tabular}{|c|c|c|c|c|c|c|}
\hline \multirow[t]{2}{*}{ Time } & \multirow{2}{*}{$\begin{array}{l}4 \mathrm{w} \\
9 \mathrm{AM}\end{array}$} & \multirow{2}{*}{$\begin{array}{l}8 \mathrm{w} \\
9 \mathrm{AM}\end{array}$} & \multicolumn{3}{|l|}{$12 \mathrm{w}$} & \multirow{2}{*}{$\begin{array}{l}\text { Pooled } \\
9 \mathrm{AM}\end{array}$} \\
\hline & & & $9 \mathrm{AM}$ & 1 PM & $5 \mathrm{PM}$ & \\
\hline$n$ & 30 & 30 & 30 & 30 & 30 & \\
\hline $\begin{array}{l}\Delta \mathrm{IOP} \\
\quad(\mathrm{mmHg})\end{array}$ & -3.2 & -3.3 & -3.6 & -3.1 & -2.9 & -3.4 \\
\hline$P$ value* & $<0.0001$ & $<0.0001$ & $<0.0001$ & $<0.0001$ & $<0.0001$ & $<0.0001$ \\
\hline $95 \% \mathrm{CI}$ & -3.8 to -2.6 & -4.0 to -2.7 & -4.2 to -3.0 & -3.7 to -2.5 & -3.5 to -2.3 & -3.9 to -2.9 \\
\hline$\% \Delta \mathrm{IOP}(\%)$ & -19.2 & -20.0 & -21.6 & -19.2 & -17.9 & -20.3 \\
\hline$P$ value* & $<0.0001$ & $<0.0001$ & $<0.0001$ & $<0.0001$ & $<0.0001$ & $<0.0001$ \\
\hline $95 \% \mathrm{CI}$ & -22.8 to -15.6 & -23.7 to -16.4 & -25.0 to -18.2 & -22.6 to -15.8 & -21.3 to -14.5 & -23.3 to 17.3 \\
\hline $\mathrm{IOP}(\mathrm{mmHg})$ & 13.4 & 13.2 & 13.0 & 12.7 & 12.8 & 13.2 \\
\hline $95 \%$ CI & 12.7 to 14.0 & 12.5 to 13.9 & 12.3 to 13.6 & 12.0 to 13.3 & 12.2 to 13.4 & 12.6 to 13.8 \\
\hline
\end{tabular}

$\triangle I O P$ IOP change, $C I$ confidence interval, IOP intraocular pressure, $\triangle I O P$ IOP change, \% $\triangle I O P$ IOP percent change, $n$ number, $w$ week

${ }^{*} t$ test

(mean $\pm \mathrm{SD}$ ) were $0.1 \pm 0.2$ at week $4,0.1 \pm 0.3$ at week 8 , and $0.3 \pm 0.5$ at week 12 ; the change reached a statistically significant level at week $12(P=0.0015)$ but not at other visits $(P=0.1607$ at week 4 and $P=0.0831$ at week 8). The changes in palpebral pigmentation scores from baseline were $0.0 \pm 0.2$ at week 4 $(P=0.3253), 0.1 \pm 0.3$ at week $8(P=0.0831)$, and $0.1 \pm 0.3$ at week $12(P=0.0831)$. The changes in sunken eyelid scores from baseline were $0.0 \pm 0.2$ at week $4(P=0.3253), 0.1 \pm 0.3$ at week $8(P=0.0831)$, and $0.1 \pm 0.3$ at week 12 $(P=0.0831)$ at week 12 . No clinically significant adverse event was reported during the study.

\section{DISCUSSION}

The purpose of this study was to confirm the sustainability of the IOP-lowering effects of travoprost $0.004 \%$ during the day in subjects with NTG. The primary endpoint was not IOP-lowering values at each measurement time but the single pooled mean of IOP change (corresponding to the average of means at each time point). To assess longitudinal IOP reduction, one approach is to perform statistical testing (e.g., $t$ test) at every measurement point and adjust multiplicity by, for example, the Bonferroni method. However, this kind of method has insufficient power to detect effects. To improve statistical precision and power, statistical inference should be limited to the pooled values of repeated measures. If IOP did not show the expected long sustainability (e.g., wide fluctuations), the pooled mean was not an adequate parameter for IOP drug evaluation. However, the continuous effects of travoprost $0.004 \%$ could be fully expected to maintain IOP fluctuations within normal limits. Accordingly, we considered it clinically relevant to use the pooled mean as the 


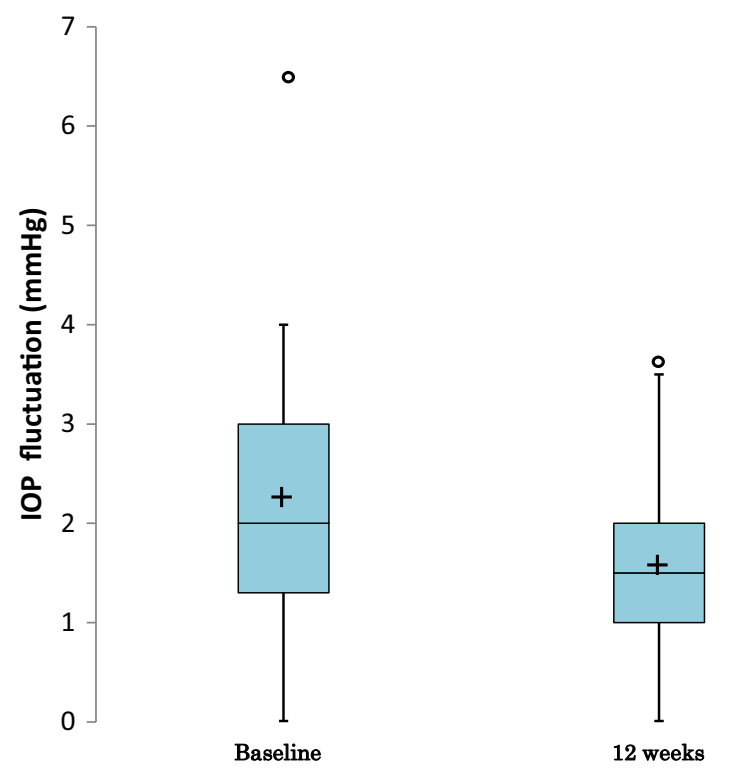

Fig. 2 Box plot of IOP fluctuation. The box shows the 75th percentile, 50th percentile (median), and 25th percentile. " + " is the mean. Whiskers show the maximum and minimum values within $1.5 \times$ the IQR from the 75 th percentile or 25 th percentile. "o" is an observation beyond $1.5 \times$ the IQR from the 75 th percentile or 25 th percentile. If no "o" exist, the whiskers are the maximum and minimum. $I O P$ intraocular pressure, $I Q R$ interquartile range

primary parameter. Therefore, we believe that the analysis plan of this study is statistically and clinically relevant.

In this study, least-square mean and CI were used for statistical inference of the pooled mean. The least-square mean is calculated based on the linear model and is usually calculated by statistical analysis software. The least-squares estimator is statistically desirable for minimizing the variance in the estimator [25]. The least-square mean of pooled mean is an average of mean values. As a result, in cases when the number of subjects is different at each time point, the least-square mean differs from values calculated from simple arithmetic means obtained from all time points. If the missing data rate is high or the difference in the missing data rates is large between time points, the
Table 4 Superficial punctate keratopathy scores

\begin{tabular}{|c|c|c|c|c|}
\hline & Baseline & $4 w$ & $8 \mathrm{w}$ & $12 \mathrm{w}$ \\
\hline Central & $0.2 \pm 0.4$ & $0.2 \pm 0.4$ & $0.0 \pm 0.2$ & $0.1 \pm 0.3$ \\
\hline Range & $(0-1)$ & $(0-1)$ & $(0-1)$ & $(0-1)$ \\
\hline$P$ value* & & 1.0000 & 0.1033 & 0.4232 \\
\hline Temporal & $0.1 \pm 0.3$ & $0.1 \pm 0.2$ & $0.0 \pm 0.0$ & $0.0 \pm 0.2$ \\
\hline Range & $(0-1)$ & $(0-1)$ & $(0-0)$ & $(0-1)$ \\
\hline$P$ value* & & 0.5722 & 0.0831 & 0.3253 \\
\hline Superior & $0.0 \pm 0.0$ & $0.0 \pm 0.0$ & $0.0 \pm 0.0$ & $0.0 \pm 0.0$ \\
\hline Range & $(0-0)$ & $(0-0)$ & $(0-0)$ & $(0-0)$ \\
\hline$P$ value* & & - & - & - \\
\hline Nasal & $0.1 \pm 0.3$ & $0.2 \pm 0.6$ & $0.1 \pm 0.3$ & $0.3 \pm 0.6$ \\
\hline Range & $(0-1)$ & $(0-2)$ & $(0-1)$ & $(0-2)$ \\
\hline$P$ value* & & 0.2547 & 0.7120 & 0.1339 \\
\hline Inferior & $0.4 \pm 0.6$ & $0.3 \pm 0.5$ & $0.2 \pm 0.5$ & $0.3 \pm 0.4$ \\
\hline Range & $(0-2)$ & $(0-1)$ & $(0-2)$ & $(0-1)$ \\
\hline$P$ value* & & 0.4232 & 0.2547 & 0.3253 \\
\hline Total & $0.7 \pm 1.3$ & $0.7 \pm 1.3$ & $0.4 \pm 0.7$ & $0.6 \pm 1.1$ \\
\hline Range & $(0-5)$ & $(0-5)$ & $(0-3)$ & $(0-4)$ \\
\hline$P$ value* & & 0.8844 & 0.1858 & 0.7866 \\
\hline
\end{tabular}

${ }^{*} t$ test, vs. baseline

$w$ week

clinical validity of analysis results might be questionable and a missing data mechanism should be explored. However, in this study, the number of cases was almost equal at each time point. This also supports the concept that it is adequate to use least-square mean as a pooled mean.

The primary measurements objective, the least-squared mean of pooled IOPs at $9 \mathrm{AM}$ at weeks 4,8 , and 12 was $13.2 \mathrm{mmHg}$, and the difference from baseline was $-3.4 \mathrm{mmHg}$, indicating statistically and clinically significant IOP reduction efficacy $(P<0.0001)$. The pooled IOP at $9 \mathrm{AM}$ was equivalent to an average of the mean IOP values at $9 \mathrm{AM}$ at weeks 4, 8, and 12 . 
Table 5 Conjunctival hyperemia incidence

\begin{tabular}{lllll}
\hline & Baseline & $\mathbf{4} \mathbf{w}$ & $\mathbf{8} \mathbf{w}$ & $\mathbf{1 2} \mathbf{w}$ \\
\hline Conjunctival hyperemia score & $0.3 \pm 0.5$ & $0.6 \pm 0.6$ & $0.5 \pm 0.6$ & $0.6 \pm 0.7$ \\
Range & $(0-2)$ & $(0-2)$ & $(0-2)$ & $(0-2)$ \\
Eyes with score 0, $n(\%)$ & $22 / 30(73.3 \%)$ & $14 / 31(45.2 \%)$ & $17 / 31(54.8 \%)$ & $16 / 31(51.6 \%)$ \\
$P$ value $^{*}$ & & 0.0169 & 0.0573 & 0.0323 \\
\hline
\end{tabular}

One subject had a missing value at baseline

$w$ week

${ }^{*} t$ test, vs. baseline

The supportive measurements outcomes were $\Delta \mathrm{IOP}$ at $9 \mathrm{AM}$ at week 4 , week 8 , and week 12 , and change rates from baseline $(\% \Delta \mathrm{IOP})$ at $1 \mathrm{PM}$ and $5 \mathrm{PM}$ at week 12 . The $\Delta \mathrm{IOPs}$ showed IOP reduction $(P<0.0001)$ at any point, and the $\% \Delta \mathrm{IOP}$ were $-19.2 \%$ and $-17.9 \%$ at $1 \mathrm{PM}$ and $5 \mathrm{PM}$ at week 12 . These results are consistent with those of a study involving NTG patients that was conducted at a single site $[17-19,26]$. In our study, which was a multi-center trial, IOP was measured three times during the day, and the results suggest that travoprost may also decrease IOP throughout the day.

As the diurnal IOP fluctuated, differences between maximum and minimum IOP values at $9 \mathrm{AM}, 1 \mathrm{PM}$, and $5 \mathrm{PM}$ at baseline and at week 12 were determined. Compared with baseline, 20 subjects had decreases in the diurnal IOP variability and 10 were unchanged or had increased diurnal IOP variability at week 12 . All of the latter subjects with baseline diurnal fluctuation of $2.0 \mathrm{mmHg}$ or less had unchanged or increased diurnal IOP changes, while the subjects with baseline fluctuation of $3.0-6.5 \mathrm{mmHg}$ had decreased values. According to some studies, the greater the IOP fluctuates throughout the day, the faster glaucoma progresses [9]. This study suggests that treatment with travoprost may suppress the diurnal IOP change in subjects with greater diurnal IOP variations. However, further study of diurnal IOP variations throughout a $24-\mathrm{h}$ period is warranted.

We have observed conjunctival hyperemia as one of the most common side effects of PG analogs. Compared with baseline, the conjunctive hyperemia score was elevated at weeks 4,8 , and 12 . However, the mean score had been 1 (minimum level) or less through the study period and no conjunctival hyperemia was reported as an adverse event.

The fluorescein staining scores of all five corneal zones at baseline in the included eyes were $0.7 \pm 1.3$ in total. The total SPK scores of the five areas were $0.7 \pm 1.3,0.4 \pm 0.7$, and $0.6 \pm 1.1$ at weeks 4,8 , and 12 , respectively, and showed no elevated scores at any point. BAC is the most commonly used preservative in ophthalmic solutions for glaucoma therapy. However, travoprost $0.004 \%$ does not contain BAC, but a zinc-containing self-preservation system (sofZia), that contains zinc chloride. Our findings indicate that travoprost $0.004 \%$ did not negatively affect the cornea.

The periocular scores for the change of eyelash, pigmentation, and sunken eyelids at week 12 were $0.3 \pm 0.5,0.1 \pm 0.3$, and $0.1 \pm 0.3$, respectively; the least favorable score for each item was 1.0. Although a significant change was not observed at week 12, continuous monitoring is advisable because medication is used long term in the treatment of glaucoma. 
This was a single-arm open-label study. In this type of study design, when evaluating the change in indicators from baseline value, a phenomenon called "regression to the mean" should be taken into consideration. This is a spurious effect; for example, if baseline values are higher than the overall mean, with or without medication, the post treatment values (or the following measurement values) tend to be closer to the overall mean. Randomized controlled trials should be used to compensate the influences of "regression to the mean" and to estimate the true drug efficacy. In the future, we need to consider further studies in this area.

\section{CONCLUSIONS}

Travoprost $0.004 \%$ solution significantly lowers mean diurnal IOP in subjects with NTG, with the effect sustainable throughout the day. The potential for travoprost to limit the diurnal fluctuations in IOP suggests a possible role in slowing the progression of glaucoma. More detailed study of the effects of travoprost on diurnal IOP variations throughout a 24 -h period is warranted.

\section{ACKNOWLEDGMENTS}

Sponsorship, the article processing charges, and the open access fee for this study were provided by Alcon Japan Ltd. The authors would like to thank Dr. Daniel McGowan on behalf of Springer Healthcare for providing English-language editing, which was funded by Alcon Japan Ltd. All named authors meet the International Committee of Medical Journal Editors (ICMJE) criteria for authorship for this manuscript, take responsibility for the integrity of the work as a whole, and have given final approval for the version to be published. They take full responsibility for the scope, direction, content, and editorial decisions relating to, the manuscript, were involved at all stages of development, and have approved the submitted manuscript.

Disclosures. Tomoko Naito has received lecture fees from Alcon Japan Ltd, Pfizer Japan Inc., Senju Pharmaceutical Co., Ltd, Santen Pharmaceutical Co., Ltd, and NIDEK Co., Ltd. Shinichi Okuma has received lecture fees from Alcon Japan Ltd, Pfizer Japan Inc., and KOWA Pharmaceutical Co., Ltd. Mikio Nagayama has received lecture fees from Alcon Japan, Senju Pharmaceutical Co., Ltd, Santen Pharmaceutical Co., Ltd, and KOWA Pharmaceutical Co., Ltd. Shiro Mizoue has received lecture fees from Alcon Japan Ltd, Pfizer Japan Inc., MSD K.K, NIDEK Co., Ltd, Senju Pharmaceutical Co., Ltd, Santen Pharmaceutical Co., Ltd, KOWA Pharmaceutical Co., Ltd, R-Tech Ueno Ltd, and Kaken Pharmaceutical Co., Ltd. Mineo Ozaki has received lecture fees from Pfizer Japan Inc. Koji Namiguchi has received lecture fees from Alcon Japan Ltd, Pfizer Japan Inc., Senju Pharmaceutical Co., Ltd, Santen Pharmaceutical Co., Ltd, and Novartis Pharma K.K. Kazuhisa Miyamoto has received lecture fees from Alcon Japan Ltd, Pfizer Japan Inc., Otsuka Pharmaceutical Co., Ltd, Senju Pharmaceutical Co., Ltd, Santen Pharmaceutical Co., Ltd, Novartis Pharma K.K. KOWA Pharmaceutical Co., Ltd, Bayer Yakuhin, Ltd, and HOYA CORPORATION. Masaki Tanito has received lecture fees from Alcon Japan Ltd, Pfizer Japan Inc., Senju Pharmaceutical Co., Ltd, Santen Pharmaceutical Co., Ltd, KOWA Pharmaceutical Co., Ltd, HOYA CORPORATION., Bayer Yakuhin, Ltd, and MSD KK. Keiji Yoshikawa has received fees for expert testimony from Santen Pharmaceutical Co., Ltd, and lecture fees from Alcon Japan Ltd, 
Senju Pharmaceutical Co., Ltd, Santen Pharmaceutical Co., Ltd, Pfizer Japan Inc., Otsuka Pharmaceutical Co., Ltd, R-Tech Ueno Ltd, Kaken Pharmaceutical Co., Ltd, KOWA Pharmaceutical Co., Ltd, and MSD KK. The authors report no other conflicts of interest related to this work.

Compliance with Ethics Guidelines. All procedures followed were in accordance with the ethical standards of the responsible committee on human experimentation (institutional and national) and with the Helsinki Declaration of 1975 , as revised in 2000 and 2008. Informed consent was obtained from all subjects for being included in the study.

Open Access. This article is distributed under the terms of the Creative Commons Attribution-NonCommercial 4.0 International License (http://creativecommons.org/licenses/ by-nc/4.0/), which permits any noncommercial use, distribution, and reproduction in any medium, provided you give appropriate credit to the original author(s) and the source, provide a link to the Creative Commons license, and indicate if changes were made.

\section{REFERENCES}

1. Iwase A, Suzuki Y, Yamamoto $\mathrm{T}$, et al. The prevalence of primary open-angle glaucoma in Japanese. The Tajimi Study. Ophthalmology. 2004;111:1641-8.

2. Yamamoto T, Iwase A, Arai M, et al. The Tajimi Study report 2: prevalence of primary angle closure and secondary glaucoma in a Japanese population. Ophthalmology. 2005;112:1661-9.

3. Japan Glaucoma Society Guidelines for Glaucoma (3rd edition). [Article in Japanese] Nihon Ganka Gakkai Zasshi 2012;116:7-46.
4. American Academy of Ophthalmology. Summary benchmarks for preferred practice pattern ${ }^{\circledR}$ guidelines. http://www.aao.org. Accessed 22 Sept 2015.

5. The AGIS Investigators. The Advanced Glaucoma Intervention Study (AGIS): 7. The relationship between control of intraocular pressure and visual field deterioration. Am J Ophthalmol. 2000;130:429-40.

6. Collaborative Normal-Tension Glaucoma Study Group. The effectiveness of intraocular pressure reduction in the treatment of normal-tension glaucoma. Collaborative Normal-Tension Glaucoma Study Group. Am J Ophthalmol. 1998;126:498-505.

7. Aoyama A, Ishida K, Sawada A, Yamamoto T. Target intraocular pressure for stability of visual field loss progression in normal-tension glaucoma. Jpn J Ophthalmol. 2010;54:117-23.

8. Naito T, Yoshikawa K, Mizoue S, et al. Relationship between progression of visual field defect and intraocular pressure in primary open-angle glaucoma. Clin Ophthalmol. 2015;9:1373-8.

9. Asrani S, Zeimer R, Wilensky J, et al. Large diurnal fluctuations in intraocular pressure are an independent risk factor in patients with glaucoma. J Glaucoma. 2000;9:134-42.

10. Caprioli J, Coleman A. Intraocular pressure fluctuation a risk factor for visual field progression at low intraocular pressures in the advanced glaucoma intervention study. Ophthalmology. 2008;115:1123-9.

11. Shin J, Lee JW, Choi BS, et al. The circadian changes of intraocular pressure and ocular perfusion pressure after tafluprost compared with travoprost in normal tension glaucoma. J Ocul Pharmacol Ther. 2014;30:803-9.

12. Netland PA, Randry T, Sullivan EK, et al. Travoprost compared with latanoprost and timolol in patients with open-angle glaucoma or ocular hypertension. Am J Ophthalmol. 2001;132:472-84.

13. Konstas AG, Kozobolis VP, Katsimpris IE, et al. Efficacy and safety of latanoprost versus travoprost in exfoliative glaucoma patients. Ophthalmology. 2007;114:653-7.

14. Dubiner HB, Sircy MD, Landry T, et al. Comparison of the diurnal ocular hypotensive efficacy of travoprost and latanoprost over a 44-hour period in patients with elevated intraocular pressure. Clin Ther. 2004;26:84-91. 
15. Quaranta L, Riva I, Katsanos A, Floriani I, Centofanti M, Konstas AG. Safety and efficacy of travoprost solution for the treatment of elevated intraocular pressure. Clin Ophthalmol. 2015;9:633-43.

16. Dubiner HB, Noecker R. Sustained intraocular pressure reduction throughout the day with travoprost ophthalmic solution $0.004 \%$. Clin Ophthalmol. 2012;6:525-31.

17. Riva I, Katsanos A, Floriani I, et al. Long-term 24-hour intraocular pressure control with travoprost monotherapy in patients with primary open-angle glaucoma. J Glaucoma. 2014;23:535-40.

18. Nomura Y, Nakakura S, Moriwaki M, Takahashi Y, Shiraki K. Effect of travoprost on 24-hour intraocular pressure in normal tension glaucoma. Clin Ophthalmol. 2010;4:643-7.

19. Seibold LK, Kahook MY. The diurnal and nocturnal effects of travoprost in normal-tension glaucoma. Clin Ophthalmol. 2014;8:2189-93.

20. David AA, Robert JN, Malik YK. Effects of benzalkonium chloride-preserved, polyquad-preserved, and sofZia-preserved topical glaucoma medications on human ocular epithelial cells. Adv Ther. 2010;27:837-45.
21. Lewis RA, Katz G, Weiss MJ, et al. Travoprost BAC-free Group. Travoprost $0.004 \%$ with and without benzalkonium chloride: a comparison of safety and efficacy. J Glaucoma. 2007;16(1):98-103.

22. Lemp MA. Report of the National Eye Institute/ industry workshop on Clinical Trials in Dry Eyes. CLAO J. 1995;21:221-32.

23. Yamazaki S, Nanno M, Kimura T, Suzumura $H$, Yoshikawa K. Effects of switching to SofZia-preserved travoprost in patients who presented with superficial punctate keratopathy while under treatment with latanoprost. Jpn J Ophthalmol. 2010;54:7-14.

24. Mizoue S, Nakano T, Fuse N, Iwase A, Matsumoto S, Yoshikawa K. Travoprost with sofZia ${ }^{\circledR}$ preservative system lowered intraocular pressure of Japanese normal tension glaucoma with minimal side effects. Clin Ophthalmol. 2014;8:347-54.

25. SAS Institute Inc.: SAS/STAT ${ }^{\circledR} 9.2$ User's Guide: The Mixed Procedure; 2008.

26. Ang GS, Kersey JP, Shepstone L, Broadway DC. The effect of travoprost on daytime intraocular pressure in normal tension glaucoma: a randomised controlled trial. $\mathrm{Br} \mathrm{J}$ Ophthalmol. 2008;92(8):1129-33. 